https://helda.helsinki.fi

\title{
Minimal Important Change for the Visual Analogue Scale Foot and Ankle (VAS-FA)
}

Saarinen, Antti J.

2021-02

Saarinen , A J , Uimonen , M M , Sandelin , H , Toom , A , Richter , M \& Repo , J P 2021 , ' Minimal Important Change for the Visual Analogue Scale Foot and Ankle (VAS-FA) ', Foot and Ankle Surgery, vol. 27 , no. 2 , pp. 196-200 . https://doi.org/10.1016/j.fas.2020.04.005

http://hdl.handle.net/10138/329406

https://doi.org/10.1016/j.fas.2020.04.005

acceptedVersion

Downloaded from Helda, University of Helsinki institutional repository.

This is an electronic reprint of the original article.

This reprint may differ from the original in pagination and typographic detail.

Please cite the original version. 


\section{Journal Pre-proof}

Minimal Important Change for the Visual Analogue Scale Foot and Ankle (VAS-FA)

Antti J. Saarinen, Mikko M. Uimonen, Henrik Sandelin, Alar Toom, Martinus Richter, Jussi P. Repo

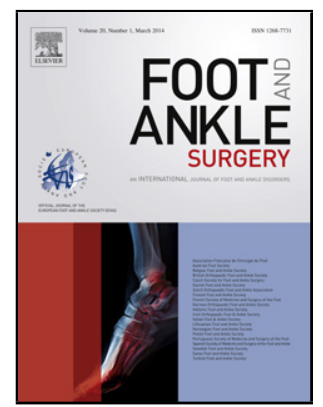

PII:

S1268-7731(20)30070-9

DOI: https://doi.org/10.1016/j.fas.2020.04.005

Reference:

FAS 1444

To appear in:

Foot and Ankle Surgery

Received Date:

11 February 2020

Revised Date:

28 March 2020

Accepted Date:

12 April 2020

Please cite this article as: $\{$ doi: https://doi.org/

This is a PDF file of an article that has undergone enhancements after acceptance, such as the addition of a cover page and metadata, and formatting for readability, but it is not yet the definitive version of record. This version will undergo additional copyediting, typesetting and review before it is published in its final form, but we are providing this version to give early visibility of the article. Please note that, during the production process, errors may be discovered which could affect the content, and all legal disclaimers that apply to the journal pertain.

(C) 2020 Published by Elsevier. 
Minimal Important Change for the Visual Analogue Scale Foot and Ankle (VAS-FA)

Antti J. Saarinen ${ }^{\mathrm{a},{ }^{*}}$, Mikko M. Uimonen ${ }^{\mathrm{b}}$, Henrik Sandelin ${ }^{\mathrm{c}, \mathrm{d}}$, Alar Toom ${ }^{\mathrm{b}}$, Martinus Richter ${ }^{\mathrm{e}}$, Jussi P. $\operatorname{Repo}^{\mathrm{b}}$

${ }^{a}$ Department of Paediatric Orthopaedic Surgery, University of Turku and Turku University Hospital, Turku, Finland

${ }^{b}$ Department of Surgery, Central Finland Hospital District, Jyväskylä, Finland.

${ }^{c}$ Department of Orthopedics and Traumatology, Helsinki University Hospital and University of Helsinki, Finland

${ }^{d}$ Orthopaedic Department, Liverpool Hospital, Sydney, New South Wales, Australia

${ }^{\mathrm{e}}$ Department for Foot and Ankle Surgery Rummelsberg and Nuremberg, Germany

* Corresponding author at: Department of Paediatric Orthopaedic Surgery, University of Turku and Turku University Hospital Kiinamyllynkatu 4-8, 20521 Turku, Finland.

E-mail address: antti.j.saarinen@utu.fi (A. Saarinen). 


\section{Highlights}

- At least 139 patient reported outcome measures are used in foot and ankle surgery

- There is a notable variance in reliability and validity in the outcome measures

- Minimal Important Change is important in interpreting outcome measure results

- VAS-FA was found to efficiently detect changes in patients undergoing surgery

\section{Abstract (150 words)}

Background: Visual analogue scale foot and ankle (VAS-FA) is a patient-reported outcome measure for foot and ankle disorders. The VAS-FA is validated into several languages and well adopted into use. Nonetheless, minimal important change (MIC) for the VAS-FA has not been estimated thus far. Methods: The VAS-FA score was obtained from 106 patients undergoing surgery for various foot and ankle complaints. MIC was estimated using an anchor-based predictive method.

Results: The adjusted MIC was 6.8 for total VAS-FA score, and 9.3 for the Pain, 5.8 for the Function, and 5.7 for the Other complaints subscales. The VAS-FA score was found to separate improvement and deterioration in patients' foot and ankle condition.

Conclusions: MIC was successfully defined for the VAS-FA in the current study. The VAS-FA can be used to evaluate foot and ankle patients' clinical foot and ankle status and its change. Further research on estimating disease-specific MICs is recommended.

Keywords: minimal clinical change; patient reported outcome measure; foot; ankle; VAS-FA 


\section{Introduction}

Patient-reported outcome measures (PROMs) are clinical outcome assessment tools used to measure subjective symptoms and the effect of treatment to patients' health ${ }^{1}$. Ideal assessment instrument is responsive to clinical change, reliable, validated, and its scores are repeatable ${ }^{2}$. Objective measures, such as radiographs, may not indicate relevant clinical change in function, health-related quality of life or pain.

In orthopaedic surgery, use of PROMs has become more common in recent years ${ }^{3}$. At least 139 clinical outcome assessment scales have been used in foot and ankle surgery ${ }^{1}$. Majority of foot and ankle specialists are reported to use PROMs in their daily work ${ }^{3}$. There is a notable variance in evidence of validity and reliability of scores obtained using different foot and ankle questionnaires ${ }^{4}$. Validated disease-specific and generic instruments are recommended for evaluating treatment outcomes as they can provide valuable information which cannot be obtained in other methods ${ }^{5}$. Nonetheless, there is no clear consensus on the recommendation on which PROMs to use for foot and ankle patients. Therefore, more evidence on the measurement properties of foot and ankle PROMs is needed ${ }^{1}$.

The Visual analogue scale foot and ankle (VAS-FA) has been designed to evaluate the subjective outcomes after foot and ankle surgery ${ }^{6}$. It has been validated for both healthy individuals and patients with foot and ankle pathologies ${ }^{67}$. The VAS-FA consists of 20 items completed on a visual analogue scale $^{7}$. Visual analogue scale is known to be easy to use, reliable, and has been validated for pain assessment ${ }^{8}$. The items can be divided into three subscales, including pain, function, and other complaints. The score can be calculated in case of missing answers. The instrument has been well adopted by the orthopaedic foot and ankle community and translated into several languages ${ }^{3} 91011$. The VAS-FA has been reported to compare favorably with other foot and ankle PROMs, such as the Lower extremity functional scale (LEFS), the Western Ontario and McMaster Universities osteoarthritis index (WOMAC), and the American Orthopaedic Foot and Ankle Society (AOFAS) scale ${ }^{10}{ }^{12}$. Although the 
VAS-FA instrument is widely tested for its psychometric properties, the minimal important change (MIC) has not been estimated for the VAS-FA thus far.

MIC can be used to determine clinically significant change from subjective patient-reported score ${ }^{13}$. Although statistical significance is useful in interpretation of change, it is closely connected to the sample size and does not interpret meaningful clinical change in PROM results ${ }^{14}$. The MIC can be implemented in both clinical and scientific work. MIC indicates the smallest change with clinical meaning in outcome assessment or follow-up. Changes greater than MIC are considered clinically relevant, indicating meaningful change in patient-experienced health status.

The purpose of the present study is to estimate the MIC of VAS-FA using an anchor-based predictive modeling method.

\section{Methods}

Ethics Committee of the Hospital District of Helsinki and Uusimaa approved the study protocol. Patients were recruited to the study face-to-face or via mail before surgery. Patients completed the validated Finnish version ${ }^{9}$ of VAS-FA either electronically on a tablet computer, or as a paper-and-pen version ${ }^{15}$. Written consent in accordance with the principles of the Declaration of Helsinki was obtained from participants. Study inclusion criteria were as follows: at least 18 years old, full understanding of Finnish, coherence in participating in the study, and a preset plan to conduct elective foot or ankle surgery by an orthopedic surgeon. Patients who did not meet the inclusion criteria were excluded from the study. Patients who had completed the VAS-FA on two different occasions with a 6-months interval were included in the final analysis. At the time of the second administration of the VAS-FA, the patients also responded to an anchor item: "How do you rate your current foot or ankle status compared to the status before surgery?". The anchor item had response categories on a five-level Likert scale from "Notably 
impaired" to "Notably improved". This anchor was designed to capture whether the patients' selfperceived situation of the foot or ankle was improved or deteriorated compared to the situation before surgery. It was used as an external reference for improvement was used to measure the true change that the patients considered as meaningful.

\subsection{Statistical methods}

Data is presented as means with standard deviation (SD), medians with interquartile ranges (IQR), numbers with percentages, or $95 \%$ confidence intervals $(95 \% \mathrm{CI})$.

The VAS-FA total score as well as subscale scores were analyzed independently. Patients with complete data on VAS-FA scores in $1^{\text {st }}$ and $2^{\text {nd }}$ administrations as well as in the anchor item were included to the analysis. To examine the relevancy of the anchor item, the VAS-FA total and the score distributions of subscales were examined in five subgroups defined by the anchor item response categories. If average VAS-FA scores were ordered in accordance with the anchor item response category, the anchor question was considered as relevant.

In order to determine the MIC values for VAS-FA and the subscales, the patients were divided into improved and not improved groups using the anchor question answers. The patients that reported that their foot had "Notably improved" or "Improved" were categorized as "Improved" while the others were categorized as "Not improved". Differences in the VAS-FA total and the subscale scores between the "Improved" and "Not improved" patients were investigated using independent samples t-test. Pvalues below 0.05 were considered as statistically significant.

Predictive MIC calculation method was applied in determination of the MIC for the VAS-FA as well as for the subscales. Logistic regression models were created with the VAS-FA score change between the administrations as an independent variable and reported improvement as a dependent variable. The 
proportion of the "Improved" patients was used as a pre-test probability of improvement. The MIC values were extracted together with the $95 \%$ confidence intervals (CI) for VAS-FA total score and the subscales. Furthermore, the MIC values were adjusted to the proportion of improved patients in the sample. The calculation methods have been described elsewhere in more detail ${ }^{16}{ }^{17}$. R 3.1.6 statistical software was used in the statistical analysis ${ }^{18}$. Calculations and statistical modeling were performed using the base package of R and "tidyverse" package was used in data manipulation and visualization ${ }^{19}$.

\section{Results}

A total of 106 patients provided complete data regarding both administrations VAS-FA scores and the anchor question. Majority of the patients were female (75\%, Table 1). The age distribution was wide, 22 to 80 years with the mean age of 56 years. The mean duration of symptoms before surgery was relatively long, 7.8 years. Forty-six (43\%) of the patients had undergone previous surgery. The three most common indications for surgery were hallux valgus $(n=25)$, mallet toe $(n=15)$, and hallux rigidus $(\mathrm{n}=10$, Table II). Two most common procedures were fusion of first tarsometatarsal joint $(\mathrm{n}=$ 28 ), and osteotomy of first metatarsal or tarsal bone $(\mathrm{n}=13$, Table II).

The average VAS-FA total and subscale scores were ordered according to the anchor item response categories of "Notably improved" to "Impaired" (Figures I - IV). In the "Notably impaired" category, the mean VAS-FA scores indicated towards better outcome than in "Impaired" category in the VASFA total and all subscales.

After dividing the patients into subgroups of "Improved" and "Not improved" patients, a total of $83 \%$ of the patients were categorized into improved subgroup. The mean increase in the VAS-FA scores of the improved patients was higher than those of the not improved patients regarding the total and all subscales (Table III). The pre-test probability of 0.83 was used in estimation of MIC for the VAS-FA 
and each of its subscale (Figures V - VIII). The adjustment for the proportion of improved patients reduced the MIC values (Table III).

\section{Discussion}

The adjusted MIC values of VAS-FA were similar for the overall score (6.8), and the subscales Function (5.8) and Other complaints (5.7), and slightly larger in Pain subscale (9.3) (Table III). In every subscale and the overall score, the MIC defined in the present study was greater than the minimal detectable change reported in a previous study ${ }^{9}$, indicating relevant results. According to the findings of the present study, the VAS-FA effectively separates between improved and unimproved patients.

Patient-experienced health is more difficult to measure than traditional measures, such as radiographic findings. Successful treatment aims to increase the health benefit using different health care modalities. The use of PROMs in clinical orthopaedic trials has increased in the recent years, leading to more research done on PROM scores ${ }^{2}$. The most important properties of PROMs are thought to be validity, reliability, and responsiveness ${ }^{20}$, which have been previously reported for the VAS-FA ${ }^{911}{ }^{12}$. In addition to these, defining the MIC is important for clinical use of PROM to understand the clinical interpretation of score results ${ }^{13}$, i.e. to distinguish weather results are, not only statistically significant, but more importantly also clinically relevant.

Estimating the significance of PROM answers in clinical work requires the knowledge of the measurement properties of PROM scores, including MIC. In scientific work, MIC provides additional use for calculating the study power for prospective studies. Change in the points of PROMs can also be used as an endpoint in scientific studies, and only differences greater than MIC should steer decisions to change clinical practice ${ }^{21}$. In clinical practice, knowing the MIC of an outcome measure can provide information on the effect of symptoms or their treatment to patient's subjective quality of life, function, 
or pain etc ${ }^{21}$. Change greater than the MIC, for example after surgical treatment, indicates meaningful treatment effect. Further, MIC can be used for screening patients in outpatient control visits for the progress of rehabilitation. Knowing the MIC helps to estimate the clinical gains of a treatment and can be used to demonstrate longitudinal treatment effect to the patient when different treatment options are discussed.

Various statistical methods are used to determinate the MIC, including the distribution-based and anchor-based methods ${ }^{22}$. Distribution-methods utilize statistical distribution and mean score values ${ }^{23}$. Anchor-based methods study the relation of external anchor-question and the score values ${ }^{24}$. In the present study, the anchor-based predictive method described by Terluin et al. was used ${ }^{16}$. Predictive modeling method has been reported to be more precise when compared to another often-used method, the receiver operating characteristic (ROC) analysis ${ }^{16}$. Furthermore, adjustment of the MIC values to the proportion of the improved have been proposed to improve the accuracy of the MIC estimates ${ }^{17}$.

Despite not being validated and holding several pitfalls, American Orthopaedic Foot and Ankle Society (AOFAS) score is the most commonly used foot and ankle score ${ }^{3}$. The AOFAS Research committee suggests using another PROM than the AOFAS score and states some of its weaknesses: limited precision, difficultly interpretable questions, unreliability, and low levels of correlation when compared to other validated PROMs ${ }^{25}$. The recently introduced EFAS score is a six-item PROM that was developed by the Score committee of European Foot and Ankle Society ${ }^{26}$. The EFAS score addresses the need of language specific validation, and it has been validated in several European languages. Further research including defining the MIC for the EFAS score is warranted in the future. In their comprehensive systematic review, Jia et al. identified 115 studies on the measurement properties of 50 different PROMs ${ }^{4}$. Their review found limited scientific evidence of psychometric (measurement) properties on majority of the scores. MIC has been previously defined to at least the following foot and 
ankle scores: the Manchester oxford foot questionnaire (MOXFQ) ${ }^{27}$, the Foot and ankle ability measure $(\text { FAAM })^{28}$, the VISA-A ${ }^{29}$, the AOFAS ${ }^{30}$, the Ankle Osteoarthritis Scale $(\mathrm{AOS})^{31}$, the Lower extremity functional scale (LEFS) ${ }^{32}$, Self-reported foot and ankle score (SEFAS) ${ }^{33}$. A small portion of foot and ankle PROMs have had their MIC estimated. The number of foot and Ankle PROMs should however be limited, as MIC for each PROM need to be estimated for the most common and important conditions that foot and ankle surgeons treat. Thus, further scientific evidence of measurement properties is needed to compare and to decide on recommendable foot and ankle score.

The current study was conducted with a representative sample of foot and ankle patients that received operative treatment. The limitations of the present study are relatively wide range of clinical diagnoses which on one hand reflects positively on the generalizability of the results and on the other hand does not specify the MICs for a distinct foot or ankle patient group. In the future, MIC for the VAS-FA can be defined specifically for a certain foot and ankle disorder. Nonetheless, the results of the present study can be used to interpret patients' scores for relevance in score change in clinical and research settings. In the future prospective research, the authors recommend using anchor-questions in addition of VAS-FA and to analyze the data to increase the body of evidence of the MICs for the VAS-FA instrument.

\section{Conclusions}

The VAS-FA efficiently detects change in patients undergoing surgery for foot and ankle disorders. With estimation of the MIC for the VAS-FA, the score can be recommended for use of obtaining information of the clinical change in function, pain and other complaints in patients having undergone surgery for foot and ankle disorders. 


\section{Conflict of Interest Statement}

Antti J. Saarinen: nothing to disclose.

Mikko M. Uimonen: nothing to disclose.

Henrik Sandelin: nothing to disclose.

Alar Toom: nothing to disclose.

Martinus Richter: nothing to disclose.

Jussi P. Repo: nothing to disclose.

\section{References}

1. Hunt KJ, Hurwit D. Use of patient-reported outcome measures in foot and ankle research. JBJS. 2013;95:e118.

2. Smith MV, Klein SE, Clohisy JC, Baca GR, Brophy RH, Wright RW. Lower extremity-specific measures of disability and outcomes in orthopaedic surgery. JBJS. 2012;94:468-477.

3. Zwiers R, Weel H, Mallee WH, et al. Large variation in use of patient-reported outcome measures: A survey of 188 foot and ankle surgeons. Foot and Ankle Surgery. 2018;24:246-251.

4. Jia Y, Huang H, Gagnier JJ. A systematic review of measurement properties of patient-reported outcome measures for use in patients with foot or ankle diseases. Quality of Life Research. 2017;26:1969-2010.

5. Rolfson O, Eresian KC, Bohm E, et al. Patient-reported outcome measures in arthroplasty registries. Acta orthopaedica. 2016;87:3-8.

6. Richter M, Zech S, Geerling J, Frink M, Knobloch K, Krettek C. A new foot and ankle outcome score: Questionnaire based, subjective, visual-analogue-scale, validated and computerized. Foot and ankle surgery. 2006;12:191-199.

7. Stüber J, Zech S, Bay R, Qazzaz A, Richter M. Normative data of the visual analogue scale foot and ankle (VAS FA) for pathological conditions. Foot and Ankle Surgery. 2011;17:166-172.

8. Price DD, McGrath PA, Rafii A, Buckingham B. The validation of visual analogue scales as ratio scale measures for chronic and experimental pain. Pain. 1983;17:45-56. 
9. Repo JP, Tukiainen EJ, Roine RP, et al. Reliability and validity of the finnish version of the visual analogue scale foot and ankle (VAS-FA). Foot and Ankle Surgery. 2018;24:474-480.

10. Nair AV, Shamsuddin K, John PS, Hämäläinen JA, Kurien MA. Correlation of visual analogue scale foot and ankle (VAS-FA) to AOFAS score in malleolar fractures using indian language questionnare. Foot and Ankle Surgery. 2015;21:125-131.

11. Angthong C, Chernchujit B, Suntharapa T, Harnroongroj T. Visual analogue scale foot and ankle: Validity and reliability of thai version of the new outcome score in subjective form. Journal of the Medical Association of Thailand. 2011;94:952.

12. Ponkilainen VT, Tukiainen EJ, Uimonen MM, Häkkinen AH, Repo JP. Assessment of the structural validity of three foot and ankle specific patient-reported outcome measures. Foot and Ankle Surgery. 2019.

13. de Vet HC, Terwee CB, Ostelo RW, Beckerman H, Knol DL, Bouter LM. Minimal changes in health status questionnaires: Distinction between minimally detectable change and minimally important change. Health and quality of life outcomes. 2006;4:54.

14. Crosby RD, Kolotkin RL, Williams GR. Defining clinically meaningful change in health-related quality of life. J Clin Epidemiol. 2003;56:395-407.

15. Uimonen MM, Ponkilainen VT, Toom A, et al. Validity of five foot and ankle specific electronic patient-reported outcome (ePRO) instruments in patients undergoing elective orthopedic foot or ankle surgery. Foot and Ankle Surgery. 2020.

16. Terluin B, Eekhout I, Terwee CB, de Vet HC. Minimal important change (MIC) based on a predictive modeling approach was more precise than MIC based on ROC analysis. J Clin Epidemiol. 2015;68:1388-1396.

17. Terluin B, Eekhout I, Terwee CB. The anchor-based minimal important change, based on receiver operating characteristic analysis or predictive modeling, may need to be adjusted for the proportion of improved patients. J Clin Epidemiol. 2017;83:90-100. 
18. R Core Team. R: A language and environment for statistical computing. $\mathrm{R}$ foundation for statistical computing, vienna, austria. 2019. Available from: https://www.R-project.org/.

19. Hadley Wickham. Tidyverse: Easily install and load the 'tidyverse'. R package version 1.2.1. . Available from: https://CRAN.R-project.org/package=tidyverse.

20. Mokkink LB, Terwee CB, Stratford PW, et al. Evaluation of the methodological quality of systematic reviews of health status measurement instruments. Quality of Life Research. 2009;18:313333.

21. Revicki D, Hays RD, Cella D, Sloan J. Recommended methods for determining responsiveness and minimally important differences for patient-reported outcomes. J Clin Epidemiol. 2008;61:102-109. 22. Johnston BC, Ebrahim S, Carrasco-Labra A, et al. Minimally important difference estimates and methods: A protocol. BMJ open. 2015;5:e007953.

23. Guyatt GH, Osoba D, Wu AW, Wyrwich KW, Norman GR, Clinical Significance Consensus Meeting Group. Methods to explain the clinical significance of health status measures. 2002;77:371383.

24. Guyatt GH, Juniper EF, Walter SD, Griffith LE, Goldstein RS. Interpreting treatment effects in randomised trials. BMJ. 1998;316:690-693.

25. Pinsker E, Daniels TR. AOFAS position statement regarding the future of the AOFAS clinical rating systems. Foot \& ankle international. 2011;32:841-842.

26. Richter M, Agren P, Besse J, et al. EFAS Score-Multilingual development and validation of a patient-reported outcome measure (PROM) by the score committee of the european foot and ankle society (EFAS). Foot and Ankle Surgery. 2018;24:185-204.

27. Dawson J, Boller I, Doll H, et al. Minimally important change was estimated for the ManchesterOxford foot questionnaire after foot/ankle surgery. J Clin Epidemiol. 2014;67:697-705.

28. Martin RL, Irrgang JJ, Burdett RG, Conti SF, Swearingen JMV. Evidence of validity for the foot and ankle ability measure (FAAM). Foot \& Ankle International. 2005;26:968-983. 
29. McCormack J, Underwood F, Slaven E, Cappaert T. The minimum clinically important difference on the visa-a and lefs for patients with insertional achilles tendinopathy. International journal of sports physical therapy. 2015;10:639.

30. Chan HY, Chen JY, Zainul-Abidin S, Ying H, Koo K, Rikhraj IS. Minimal clinically important differences for american orthopaedic foot $\&$ ankle society score in hallux valgus surgery. Foot $\&$ ankle international. 2017;38:551-557.

31. Coe MP, Sutherland JM, Penner MJ, Younger A, Wing KJ. Minimal clinically important difference and the effect of clinical variables on the ankle osteoarthritis scale in surgically treated end-stage ankle arthritis. JBJS. 2015;97:818-823.

32. Binkley JM, Stratford PW, Lott SA, Riddle DL, North American Orthopaedic Rehabilitation Research Network. The lower extremity functional scale (LEFS): Scale development, measurement properties, and clinical application. Phys Ther. 1999;79:371-383.

33. Cöster MC, Nilsdotter A, Brudin L, Bremander A. Minimally important change, measurement error, and responsiveness for the self-reported foot and ankle score. Acta orthopaedica. 2017;88:300304. 
Figures I - IV. Distributions of the VAS-FA overall and subscale scores in anchor question response category subgroups. The boxes represent median and the whiskers represent interquartile range (IQR).
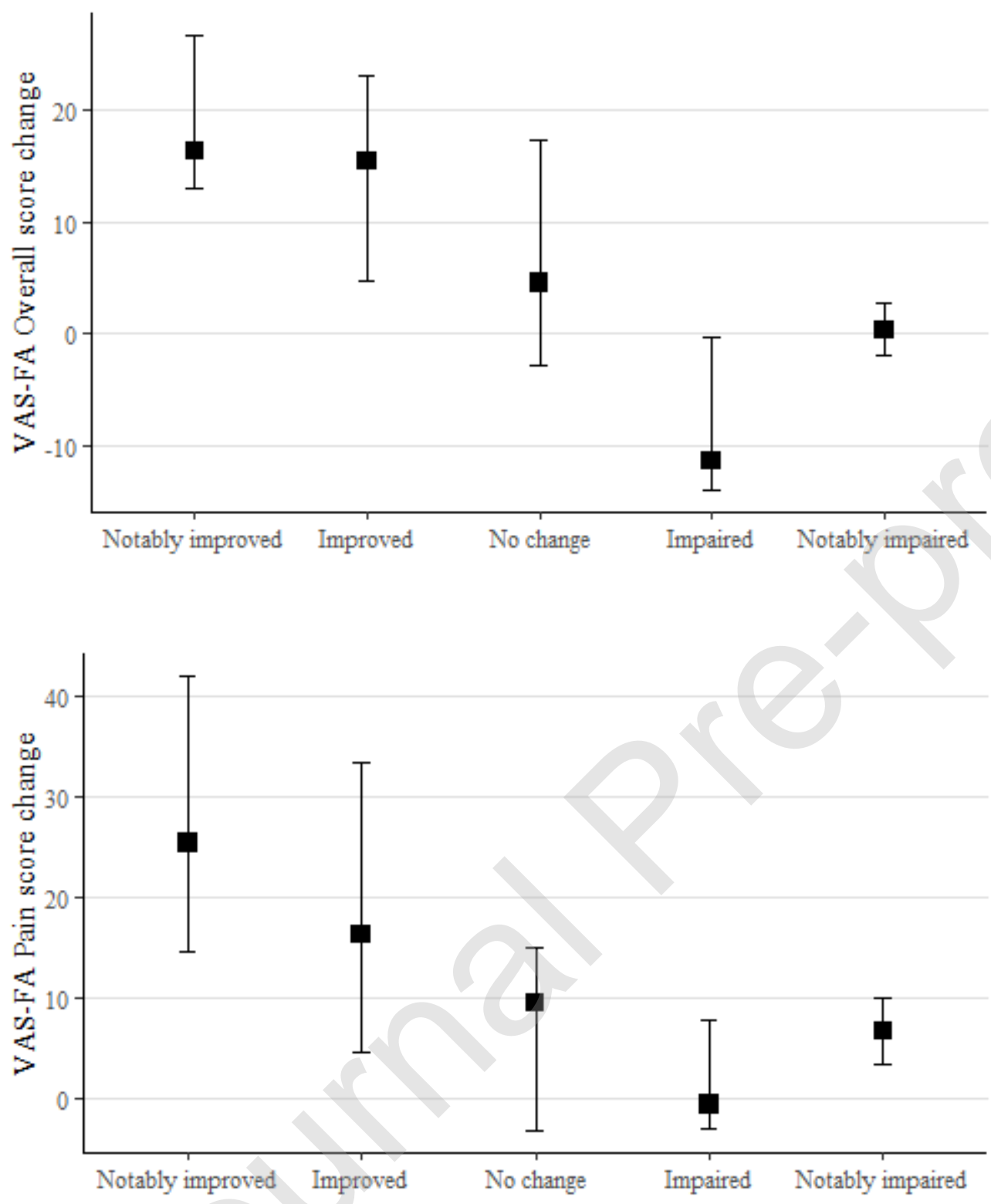

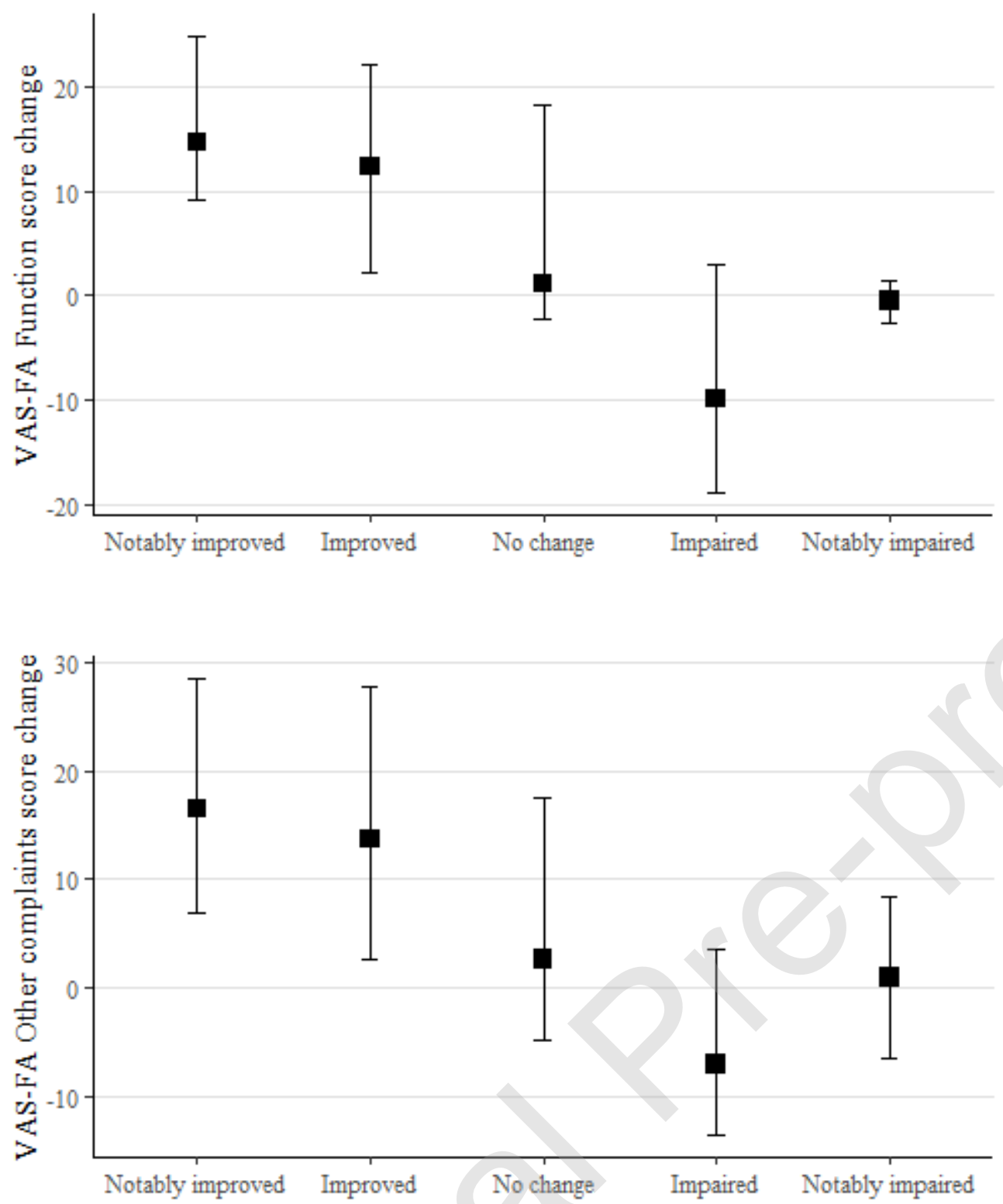
Figures V - VIII. Logistic regression models for MIC estimation. The dots on the upper and lower case illustrates the reported outcome of each patient. Pre-test probability of improvement of 0.83 was used according to the distribution of "Improved" (1.00) and "Not-improved" (0.00) patients in the sample. The MIC is estimated on the point where the two dotted lines intercept.
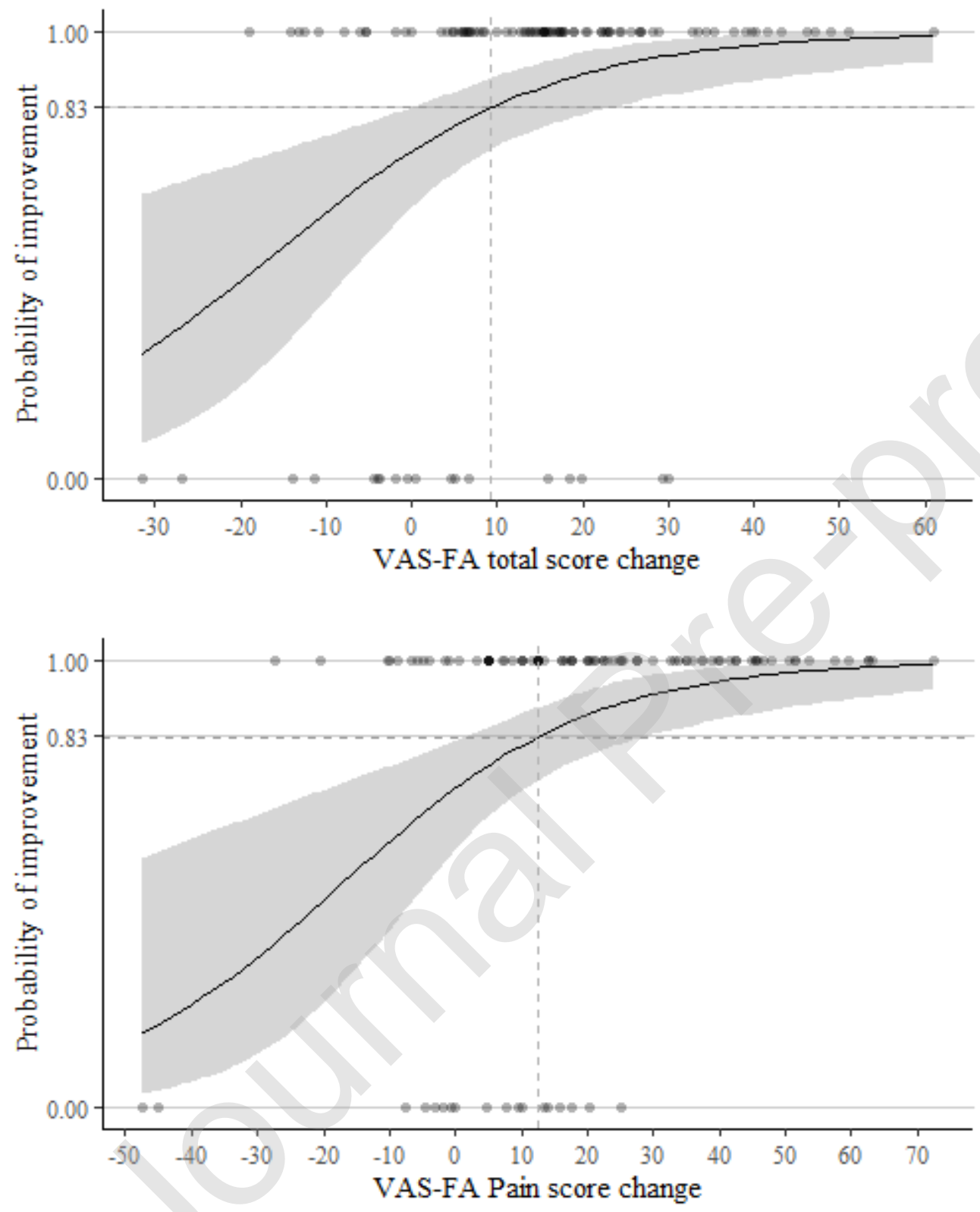

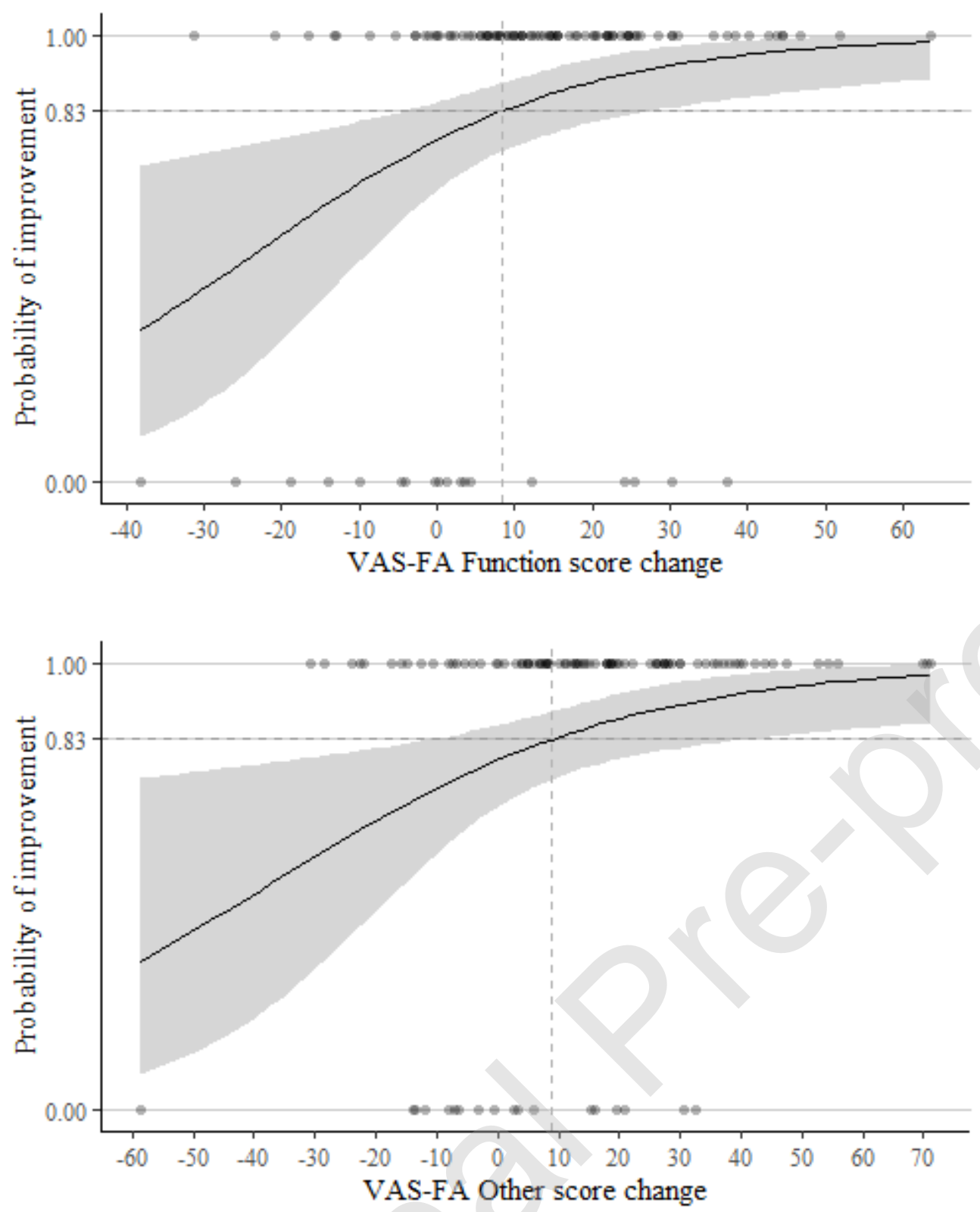

Table I. Clinical and sociodemographic characteristics.

\section{Characteristic}

Female, n $(\%)$

$79(75)$

Age (years), mean (SD, range)

$56(14,22-80)$ 
BMI, mean (SD, range)

$27(7.6,18-77)$

Education level, n (\%)

Comprehensive school 35 (33)

Upper secondary school, not graduated 5 (4.7)

Upper secondary school, graduated 10 (9.4)

Undergraduate degree, not graduated 2 (1.9)

Undergraduate degree, graduated 38 (36)

Graduate degree, not graduated 1 (1)

Graduate degree, graduated 12 (11)

Marital status, n (\%)

In a relationship

$9(8.4)$

Married

$57(54)$

Never been married

$18(17)$

Divorced

$10(9.4)$

Widow

$10(9.4)$

Duration of symptoms (years), mean (SD, range)

$7.8(9.9,0.3-50)$

Patient--reported health state, n (\%)

Excellent 2 (1.8)

Very good 21 (20)

Good 54 (51)

Moderate 24 (23)

Poor 2 (1.8)

Previous operations, n (\%)

$46(43)$

Number on previous operations, mean (SD, range)

$2(1.5,1-6)$

Patient reported comorbidities, n (\%)

Cardiovascular disease

$18(17)$

Diabetes

$11(10)$

Hypertension

$29(27)$

Rheumatic disease

$10(9.4)$

Respiratory disease

$16(15)$

Cancer

11 (10) 
Table II. Diagnoses and procedures.

\section{Diagnosis}

Hallux valgus

$\mathrm{n}(\%)$

Hallux rigidus

$24(22.6)$

Digiti malleiformis

$10(9.4)$

$8(7.5)$

Primary osteoarthritis of other joints

$6(5.7)$

Post-traumatic osteoarthritis of other joints

Fractures of lower leg

Pseudarthrosis after join fusion

Rheumatoid arthritis with rheumatoid factor

Metatarsalgia

Pain in joint

Other

\section{Procedure}

Fusion of first tarsometatarsal joint

Osteotomy or rotation osteotomy of first metatarsal or tarsal bone

Fusion of talocrural joint

Operation on fascia, ganglion, synovial sheath or bursa of ankle or foot

Removal of internal fixation device from ankle or foot

Osteotomy or rotation osteotomy of II-V metatarsal or tarsal bone

Other 
Table III. Mean score of change and MIC values for the VAS-FA.

\begin{tabular}{lcccccc}
\hline & \multicolumn{2}{c}{ Mean (SD) score change } & Sig. & & MIC (95\% CI) & Adjusted \\
& Improved & Not improved & & & & MIC \\
\hline Total & $16.7(15.6)$ & $1.8(16.9)$ & 0.002 & 9.2 & $(-3.6$ to 23.0) & 6.8 \\
Pain & $22.8(20.7)$ & $1.6(19.7)$ & $<0.001$ & 12.4 & $(-3.5$ to 29.5) & 9.3 \\
Function & $14.9(16.3)$ & $1.4(19.6)$ & 0.012 & 8.3 & $(-8.2$ to 25.1) & 5.8 \\
Other & $16.4(21.7)$ & $1.3(20.9)$ & 0.010 & 8.9 & $(-14.2$ to 33.7) & 5.7 \\
\hline \multicolumn{7}{l}{ MIC = Minimal important change; $95 \% \mathrm{CI}=95 \%$ confidence interval }
\end{tabular}

$\mathrm{MIC}=$ Minimal important change $; 95 \% \mathrm{CI}=95 \%$ confidence interval 